\title{
A High-Bandwidth MEMS Nanopositioner for On-Chip AFM: Design, Characterization, and Control
}

\author{
Mohammad Maroufi, Ali Bazaei, Member, IEEE, and S. O. Reza Moheimani, Fellow, IEEE
}

\begin{abstract}
We report the design, characterization, and control of a high-bandwidth microelectromechanical systems (MEMS) nanopositioner for on-chip atomic force microscopy (AFM). For the fabrication, a commercially available process based on silicon-on-insulator is used. The device consists of a scan table, moved in the $x-y$ plane by two sets of electrostatic comb actuators, capable of generating strokes in excess of $\pm 5 \mu \mathrm{m}$. The first resonance frequencies of the nanopositioner are approximately 4.4 and $5.3 \mathrm{kHz}$ in lateral directions. Electrothermal sensors are used to measure the displacement of the scan table. To enable fast scans, a dynamic model of the system is identified and used to design a feedback controller that damps the oscillatory behavior of the device. The nanopositioner is tested as the scanning stage of an AFM to perform high-speed scans.
\end{abstract}

Index Terms - Electrostatic actuators, electrothermal sensors, high-bandwidth nanopositioner, microelectromechanical systems (MEMS), on-chip atomic force microscopy (AFM).

\section{INTRODUCTION}

$\mathbf{T}$ $\mathrm{HE}$ ability of nanopositioners to produce high precision motions with nanometer and subnanometer resolution has led to their emergence as an integral component of instrumentation in numerous areas, such as lithography [1], scanning tunneling microscopy [2], atomic force microscopy (AFM) [3], and ultrahigh-density probe storage systems [4]-[6]. These nanopositioning stages typically have a high-positioning accuracy and a traveling speed of several hundred hertz [7], [8]. There is a significant and growing demand for high-bandwidth nanopositioning stages. For example, there is a significant push to develop high-speed AFMs that can produce real time, time-lapsed images of dynamic processes at the nanoscale. High-bandwidth nanopositioning is a critical requirement in such systems [8]-[10]. A number of meso-scale nanopositioners with satisfactory bandwidth, range, and positioning accuracy have been reported in [8] and [11]-[13].

Microelectromechanical systems (MEMS) have recently emerged as an alternative technology for the realization of nanopositioning platforms. The small size, low fabrication

Manuscript received November 28, 2013; revised April 9, 2014; accepted July 12, 2014. Date of publication August 20, 2014; date of current version February 11, 2015. Manuscript received in final form July 29, 2014. This work was supported by the Australian Research Council. Recommended by Associate Editor I. Petersen.

The authors are with the School of Electrical Engineering and Computer Science, The University of Newcastle Australia, Callaghan, NSW 2308, Australia (e-mail: mohammad.maroufi@uon.edu.au; ali.bazaei@newcastle.edu.au; reza.moheimani@newcastle.edu.au).

Color versions of one or more of the figures in this paper are available online at http://ieeexplore.ieee.org.

Digital Object Identifier 10.1109/TCST.2014.2345098 cost, and fast dynamics of MEMS nanopositioners have led to them being considered for applications, such as probe-based data storage [4], [5] and on-chip AEM [14]. The latter has promising prospects for making inexpensive AFMs widely available to the community. In this paper, we propose a MEMS device with the ultimate goal of realizing on-chip video-rate AFM.

Several MEMS nanopositioners have been reported in the literature. In [15], a high bandwidth (26 kHz), low cross coupling quasi-monolithic $X Y$ nanopositioner is proposed. Piezoeresistive sensors are incorporated in the device to allow for real-time measurement of the stage displacement in both directions. However, the total displacement range is relatively small $(\sim 5 \mu \mathrm{m})$ and a nonstandard MEMS fabrication process is required, increasing the fabrication cost. In addition, using piezoelectric actuators makes control of the device challenging due to their inherent nonlinear behavior [16]. The MEMS nanopositioners have also been proposed as an integral component of probe-based data storage systems, where an array of AFM-like cantilevers are used to store digital information as tiny indentations on a storage medium [17], [18]. A MEMS nanopositioner designed for this purpose is reported in [5]. The actuation of this device is based on voice coil motors (VCMs) that are installed through a postfabrication process. This 2-degree-of-freedom (2DOF) nanopositioner can move $\sim 120 \mu \mathrm{m}$ in either direction and has a first resonance frequency of $\sim 120 \mathrm{~Hz}$ [4], [6]. Another version of this system, in which the VCMs are replaced with electrostatic actuators, is reported in [19]. This device has half the travel range of the original positioner, but a relatively similar bandwidth.

In [14], we reported a 2DOF MEMS nanopositioner for onchip AFM. The device was fabricated through the standard SOIMUMPs process. The displacement range of this nanopositioner is more than $15 \mu \mathrm{m}$ with very low cross coupling between its two axes of motion. The design demonstrated a bandwidth of $820 \mathrm{~Hz}$, which makes it comparable with nanopositioners used in commercial AFMs. The bandwidth, however, is insufficient for high-speed AFMs. A modified version of this design equipped with displacement sensors was reported in [20]. Inclusion of sensors allowed for control of the scanner that enabled a higher scan speed. Despite this, highspeed AFM requires a nanopositioner with a wider bandwidth.

The main motivation for this paper is to design a MEMS nanopositioner that bridges the gap between regular and videorate on-chip AFM. Considering important factors, such as the achievable force from a given footprint, bandwidth, feasibility 


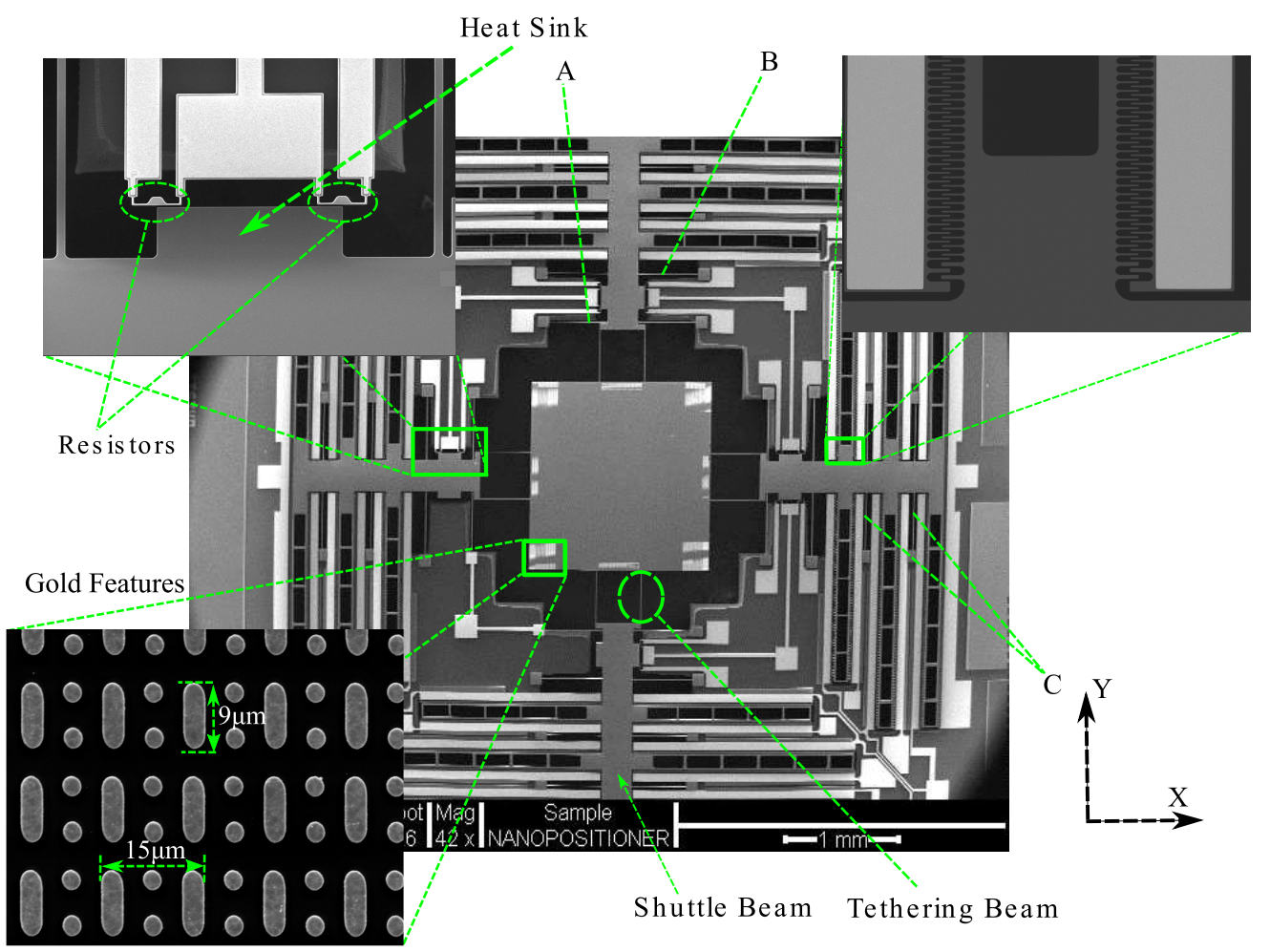

Fig. 1. MEMS $X Y$ nanopositioner. The close-up view of an electrothermal sensor with its resistors is shown in the top-left corner. In the bottom-left corner, gold features used for scanning under AFM are illustrated. The diameter of the small dots and their spacing along the $y$-axis are $3 \mu \mathrm{m}$.

of fabrication using standard processes, and linear behavior, electrostatic comb drives were selected. For each axis, a pair of differential comb drives is fabricated considerably linearizing the actuator characteristics. Electrothermal actuation is an alternative technology for MEMS nanopositioning. However, despite their small footprint and ability to produce a large force, electrothermal actuators are rather sluggish, and cannot operate at the required bandwidth [21]-[23]. Piezoelectric and electromagnetic actuation are two other possibilities. However, their incorporation into a MEMS device would require postprocessing that adds to the complexity and cost of the device [15].

To achieve a high-positioning accuracy in the presence of uncertainties, disturbances, and nonlinear effects, closedloop control is necessary. A feedback control system needs real-time measurement of the controlled variable, i.e., the position of the nanopositioner scan table. A number of displacement sensing technologies are available in MEMS. The displacement measurement method chosen here is based on the concept of electrothermal sensing [21], which is suitable for nanopositioning applications, and realizable in MEMS with a minimal footprint.

The remainder of this paper continues as follows. Section II describes the design of the MEMS nanopositioner and the tuning of the structural parameters. The electrothermal sensor and its readout circuit design are also detailed in this section. Implementation of the linear actuation mechanism is described in Section III, where the static and dynamic behavior of the device, system identification, and sensors characterization results are presented. The controller design and its implementation are addressed in Section IV. Finally, in Section V, using combined feedback/inversion-feedforward method for high-speed raster scanning, the capability of the nanopositioner for operation under an AFM is demonstrated.

\section{MEMS NANOPOSITIONER DESIGN}

Fig. 1 shows a scanning electron microscope image of the proposed 2DOF parallel kinematic mechanism stage. Electrostatic actuators on either side generate the required force which is transferred to the stage through shuttle beams. Two tethering beams are used on each side to prevent the scan table from experiencing in-plane rotations. The device was fabricated using MEMSCAP's standard SOIMUMPs process [24]. This silicon-on-insulator-based process allows a feature size of $2 \mu \mathrm{m}$ at a resolution of $0.25 \mu \mathrm{m}$ for the single crystal silicon on the device layer.

\section{A. Tuning of the Structural Parameters}

Tuning the beam parameters of the suspension systems, including their length, width, and position along the shuttle beam is an iterative task. In [25], we developed a model for the device to predict the bandwidth and displacement range of the stage. The model was used to perform the dimensional tuning through trial and error. During the design process, a number of constraints were placed on the design variables. The geometrical constraints include the limited space available on the chip, the relative position of the components, and design limitations associated with the microfabrication process, such as the maximum area allowable for the substrate and the device 
TABLE I

Geometrical and Material Properties OF THE NANOPOSITIONING STAGE

\begin{tabular}{|c|c|c|c|}
\hline Density & $2500\left(\mathrm{Kg} / \mathrm{m}^{3}\right)$ & Young's Modulus & $169(\mathrm{GPa})$ \\
\hline \hline Stage Size & $1.6 \mathrm{~mm} \times 1.6 \mathrm{~mm}$ & Thickness $(\mathrm{h})$ & $25 \mu \mathrm{m}$ \\
\hline \multirow{3}{*}{ Beams } & Beam: A & Length: $520 \mu \mathrm{m}$, Width: $9 \mu \mathrm{m}$ \\
\cline { 2 - 4 } & Beam: B & Length: $539 \mu \mathrm{m}$, Width: $9 \mu \mathrm{m}$ \\
\cline { 2 - 4 } & Beam: C & Length: $494 \mu \mathrm{m}$, Width: $6 \mu \mathrm{m}$ \\
\cline { 2 - 4 } & Tethering Beams & Length: $445 \mu \mathrm{m}$, Width: $7 \mu \mathrm{m}$ \\
\hline Fingers & \multicolumn{2}{|c|}{ Length: $20 \mu \mathrm{m}$, Width: $2 \mu \mathrm{m}$, Gap: $2 \mu \mathrm{m}$} \\
\hline \multirow{2}{*}{ Long Comb } & \multicolumn{2}{|c|}{ Length: $2007 \mu \mathrm{m}$, Width: $130 \mu \mathrm{m}$} \\
\cline { 2 - 4 } & \multicolumn{2}{|c|}{ Number of fingers in one side: 247} \\
\hline \multirow{2}{*}{ Short Comb } & \multicolumn{2}{|c|}{ Length: $1610 \mu \mathrm{m}$, Width: $128 \mu \mathrm{m}$} \\
\cline { 2 - 4 } & \multicolumn{2}{|c|}{ Number of fingers in one side: 198} \\
\hline
\end{tabular}

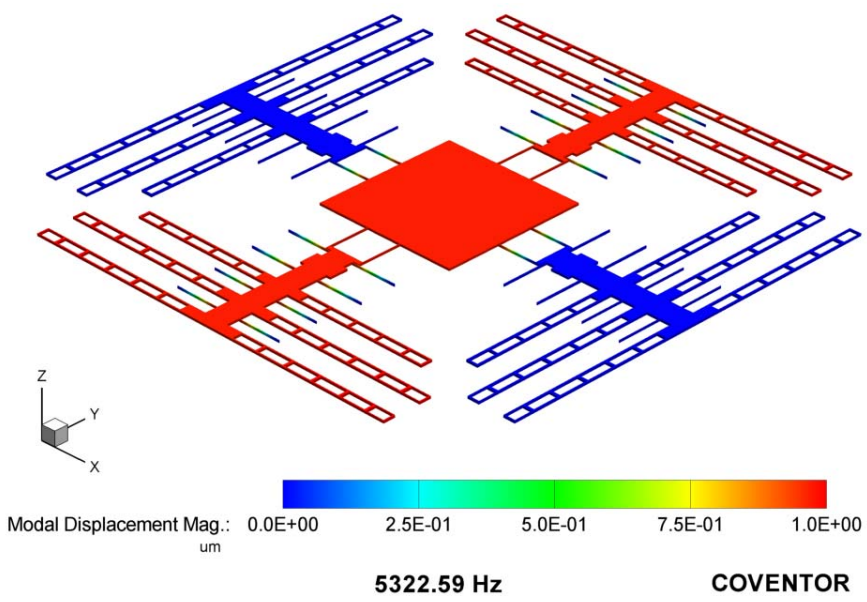

Fig. 2. First resonant mode of the nanopositioner obtained by FEM.

layer etching. In addition, the critical buckling load for the tethering beams [26] and adequate lateral stiffness for the suspensions system, to prevent the comb fingers from snap-in, are among the structural constraints [25], [27].

The maximum stress in the mechanical elements, as another design constraint, should be less than the yield stress with an appropriate margin [28]-[30]. Single crystal silicon, the structural material in SOIMUMPs process, is brittle, and its characteristic fracture strength is $\sim 1.97 \mathrm{GPa}$ with Weibull modulus of 8.9 [28]. During the design, for the yield stress, we considered a conservative value of $1 \mathrm{GPa}$.

Although the theoretical model provides an insight into the geometrical parameter values and the design characteristics, a finite element model (FEM) was also constructed to ensure that the design characteristics were acceptable. The FEM of the nanopositioner was developed using CoventorWare software. Table I presents the geometrical parameter values of the fabricated system. Fig. 2 shows the FEM simulation results of the system for the first resonant mode. Due to the symmetrical feature of the device, the same resonant mode is expected for the other axis.

Apart from the snap-in instability in the comb drive structures that should be avoided by a proper mechanical suspension system design [25], the end fingers in comb structures are also prone to side-instability [31]. Due to the asymmetric electric field, the end fingers may deflect and snap to each

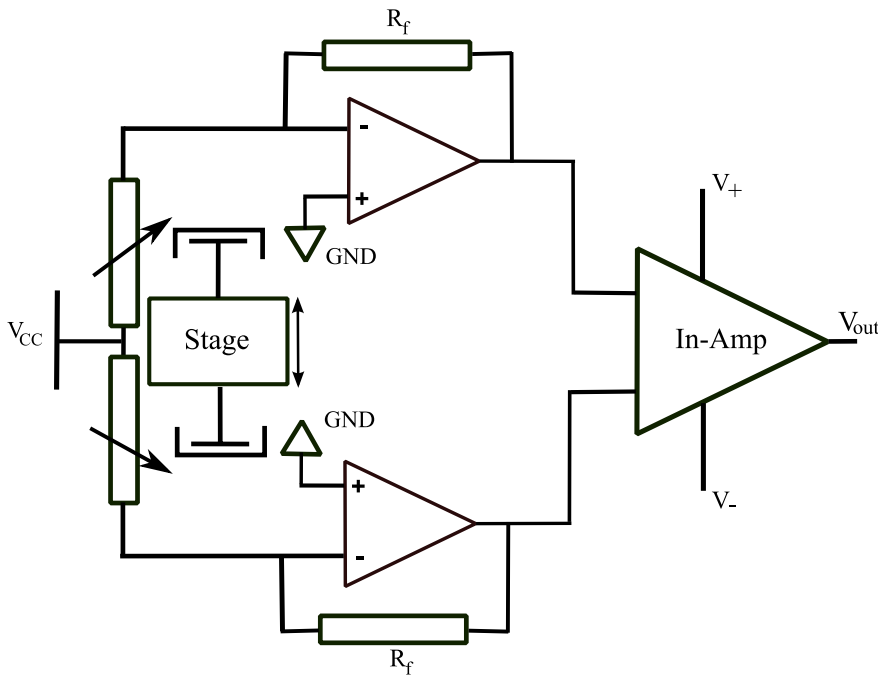

Fig. 3. Schematic view of the $I-V$ transimpedance readout circuit for the electrothermal sensors.

other at a sufficiently high voltage. As shown in Fig. 1, the end fingers are designed to be thicker compared with the remaining fingers to increase their in-plane stiffness and prevent them from experiencing this type of snap-in.

\section{B. Electrothermal Displacement Sensors}

Sensors are incorporated to measure lateral displacements of the stage. To enhance the sensitivity, linearity, and noise characteristics of the sensors, a pair of shaped beams are fabricated as position dependent resistors [32]. The beams act as heaters when biased by a sufficiently high voltage. As shown in Fig. 1, a heat sink is implemented on each shuttle beam, and its displacement changes the resistor temperatures and thereby their resistances, oppositely. The readout circuit, shown in Fig. 3, maps the resistance difference to the displacement of the heat sink. This circuit comprises two stages. In the first section, a bias voltage $\left(V_{\mathrm{cc}}\right)$ heats up the beams electrothermally and the first stage transimpedance amplifiers convert the heater currents to proportional voltages. The next stage is the differential amplifier, which amplifies the difference between the two voltages [21], [33].

Lateral movements of the heat sink (toward or away from the sensor), which adversely affect the measurement accuracy are avoided by a proper mechanical suspension design. This issue has been addressed by designing four clamped-clamped beams adjacent to each heat sink.

\section{Characterization And System Identification}

In this section, we first describe the linear actuation mechanism. Then, the characterization and system identification results are discussed. Finally, the characteristics of the electrothermal sensors and their noise characteristics are stated.

\section{A. Linear Actuation Mechanism}

The electrostatic actuation force generated by comb drives is a quadratic function of the actuation voltage $(V)$

$$
F=\frac{1}{2} \frac{\partial C}{\partial y} V^{2}=\frac{n \varepsilon_{0} h}{g} V^{2}
$$



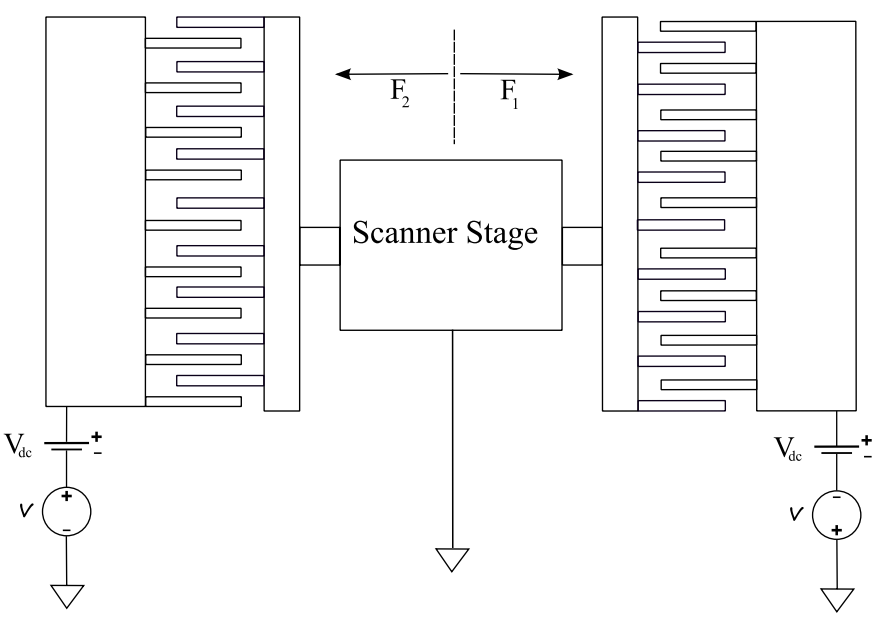

Fig. 4. Linear actuation mechanism incorporated for the electrostatic actuators.

where $n$ is the number of the fingers in one side (moving or stationary combs), $\varepsilon_{0}=8.85 \times 10^{-12} \mathrm{C}^{2} \mathrm{~N}^{-1} \mathrm{~m}^{-2}$ is the permittivity of air, $h$ is the comb finger thickness, and $g$ is the gap. Assuming a linear mechanical stiffness, the resulting displacement will also have a quadratic relation with the actuation voltage if only one of the comb drives is actuated. Avoiding such nonlinear characteristics will make the calibration and control design process more straightforward [20], [34].

To make the static voltage-displacement characteristics linear, the differential actuation method shown in Fig. 4 is used [35], [36]. In this method, the scan table and the moving combs are connected to the electrical ground, while a constant bias voltage $\left(V_{\mathrm{dc}}\right)$ plus equal and opposite voltages $v$ and $-v$ are applied to the opposing stationary combs in each direction, respectively. As an alternative method, the electrical bias voltage can be applied to the scan table and the moving combs, while the stationary combs in either side are actuated by $-v$ and $v$ [25]. However, since the nanopositioner is to be used under an AFM, electrical connection of the stage to the ground is crucial.

The net force $\left(F_{\text {net }}\right)$ exerted on the scan table by this method is calculated as

$$
F_{\text {net }}=F_{2}-F_{1}=a\left(V_{\mathrm{dc}}+v\right)^{2}-a\left(V_{\mathrm{dc}}-v\right)^{2}=4 a V_{\mathrm{dc}} v
$$

where $a=n \varepsilon_{0} h / g$ is assumed to be identical for the symmetrically fabricated comb drives. Using this approximation, the net force is linearly related to the actuation voltage $(v)$.

\section{B. Static Behavior and Sensor Calibration}

The static displacements of the stage in both axes were simultaneously measured using both Polytec MSA-050-3D and the on-chip displacement sensors. The measurements are shown in Figs. 5 and 6, respectively. The MSA uses three laser beams to measure in-plane and out of plane displacements and/or velocities of a small moving object. A dc bias voltage of $70 \mathrm{~V}$ was applied during all tests. The experiments confirm that the behavior of the device is much closer to linear compared with the alternative actuation method that results in a quadratic

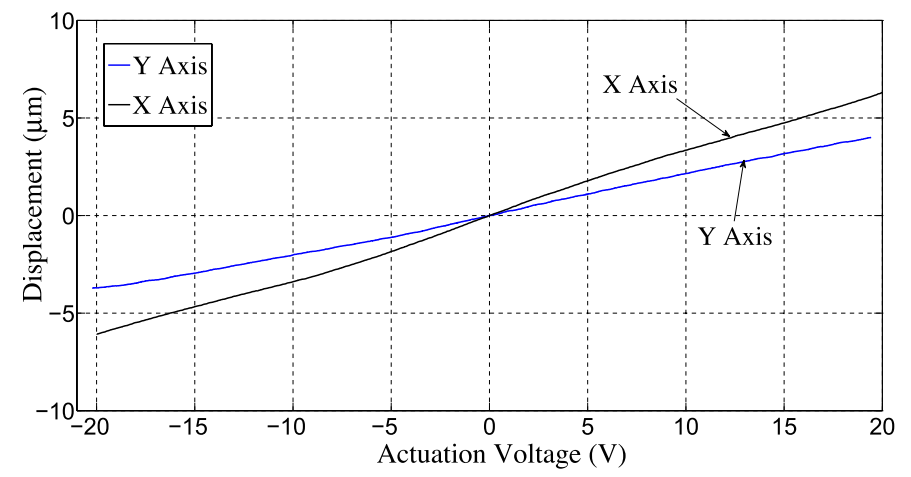

Fig. 5. Displacement of the scan table versus actuation voltage (v).

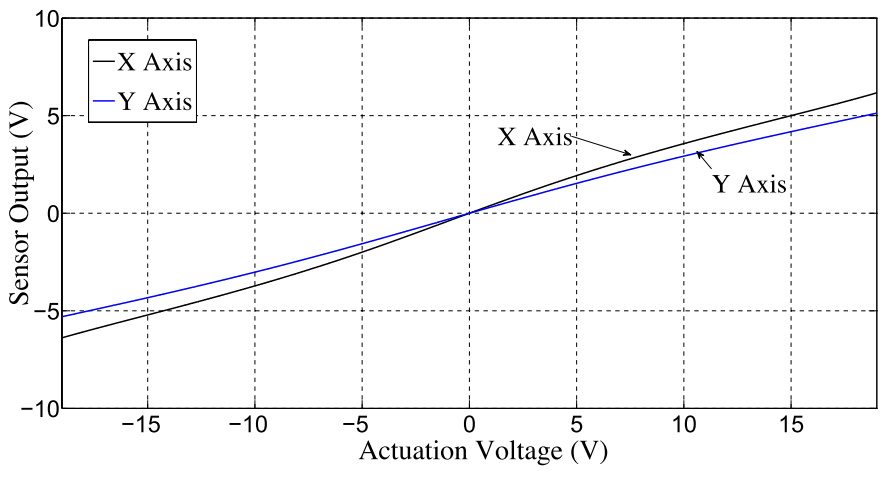

Fig. 6. Sensors output versus actuation voltage $(v)$.



Fig. 7. Schematic view of the method implemented for obtaining the open-loop frequency response of the nanopositioner using on-chip sensors.

profile [25]. In addition, displacements in excess of $\pm 5 \mu \mathrm{m}$ were achieved for both axes.

Using the experimental data shown in Figs. 5 and 6, calibration factors for $X$ and $Y$ sensors were obtained as 0.926 and $0.715 \mu \mathrm{m} / \mathrm{V}$, respectively.

\section{Frequency Response}

We obtained the open-loop frequency response of the system using both the MSA and the displacement sensors. The linear actuation mechanism with the dc bias of $70 \mathrm{~V}$ was used, and a periodic chirp signal was differentially applied to the comb drives on either side. Fig. 7 shows the schematic of the experimental setup using on-chip sensors, where an amplifier is used to convert a single ended reference signal to differential signals $v$ and $-v$ and amplify them.

Direct measurement of the displacement by the MSA shows a dominant second-order resonant behavior, as shown in Fig. 8. However, the drastic changes in magnitude and phase of the frequency response obtained by the electrothermal sensors indicate that the sensors introduce a low pass effect in the system. The resonance frequency of the stage is at $4438 \mathrm{~Hz}$ for $x$-axis and $5341 \mathrm{~Hz}$ for $y$-axis. The difference between the 

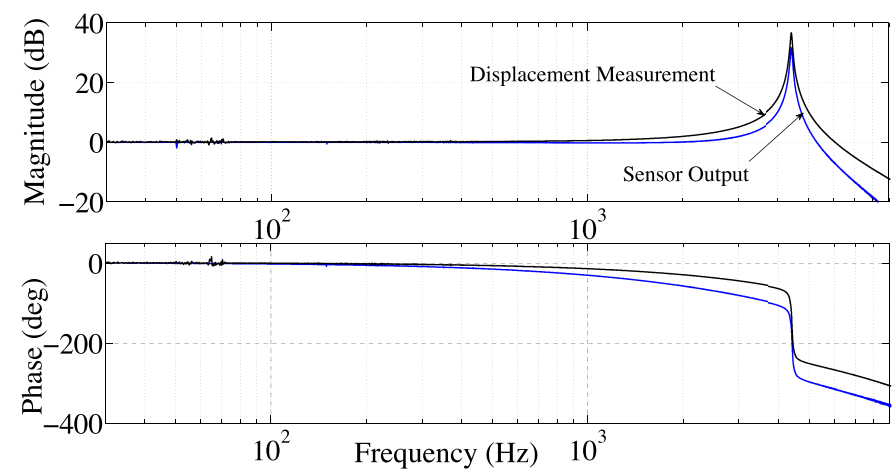

(a)
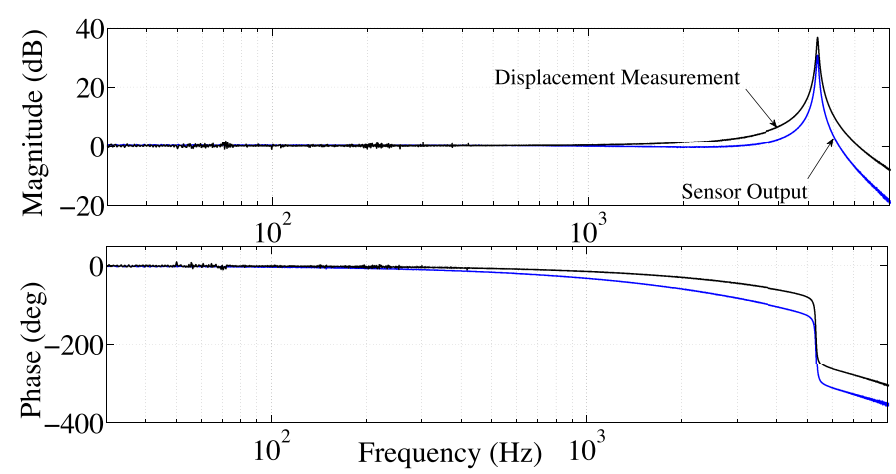

(b)

Fig. 8. Frequency responses of the system in open-loop for both axes with the MSA and the sensors. (a) $x$-axis. (b) $y$-axis.

frequency responses of the two axes is most likely due to the microfabrication tolerances.

Resonance frequencies, higher than $4.4 \mathrm{kHz}$ for both axes together with displacement ranges that exceed $\pm 5 \mu \mathrm{m}$, make this nanopositioner a promising proposition for highspeed AFM.

The amount of cross coupling measured between the two axes is about $-40 \mathrm{~dB}$. The cross coupling rejection obtained by the design is comparable and in some instances better than those reported previously which are in the range of -35 to $-40 \mathrm{~dB}[7]$.

\section{System Identification}

Considering the extremely low cross coupling between the two axes of the nanopositioner, the lateral dynamics can be accurately represented by two single-input single-output systems. Fifth-order models were fitted to the frequency domain data using least square system identification method [37]. The transfer functions are described in (3), as shown at the bottom of the page.

Due to the second-order resonant behavior of the scanner, the identified $X$ and $Y$ transfer functions have two lightly

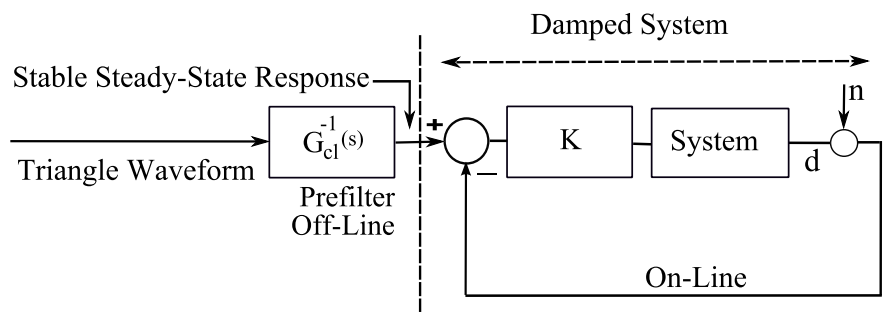

Fig. 9. Both axes of the scanner were damped using a proportional controller. To increase the bandwidth of the fast axis ( $x$-axis), inversion feedforward was used.

damped complex conjugate poles with damping ratios of 0.007 and 0.006 , respectively. There is a slow pole with a frequency of $\sim 2.8 \mathrm{kHz}$ on the real axis for both sensors, which is due to the limited bandwidth of the sensors. Each model also exhibits nonminimum phase behavior captured by a pair of conjugate right-half-plane zeros at frequencies $\sim 15 \mathrm{kHz}$. To ensure that the additional phase drop in the open-loop response when switching from the MSA to the sensor is not due to a delay in the readout circuit, we also examined the transient response of the stage in the time domain for both axes, where no delay consistent with the phase drop was observed in the sensor output.

The sensor characteristics can be estimated by the difference between the frequency response of the system with and without the sensors (Fig. 8). In [32] and [38], this type of sensor has been modeled with a first-order system. The phase drop at the resonance frequency $(4.4 \mathrm{kHz})$ is $\sim 40^{\circ}$. A first-order system with a time constant of $30 \mu$ s shows approximately the same phase drop at this frequency. This time constant is consistent with $24.4 \mu \mathrm{s}$ reported for the curved shaped electrothermal displacement sensors in [32].

\section{Design AND ImPLEMENTATION of FEEDBACK CONTROLLER}

Various methods in the literature are proposed to control the dynamics of resonant mechanical structures. These include integral resonant control [39], positive position feedback [40], and positive velocity and position feedback [41]. Most of these control methods have stability conditions that can be easily met if the plant satisfies the negative imaginary property [42], [43], which is usually satisfied for collocated resonant structures [44]. Such a property, however, does not hold for this system, since its Nyquist plot is not limited to the third and fourth quadrants.

A static gain controller $(K)$ is used to augment damping of the lightly damped modes of the nanopositioner, as shown in Fig. 9. Straightforward design, implementation, and tuning are among the advantages of this type of controller. The controller

$$
\begin{aligned}
G_{x x} & =\frac{2.785 \times 10^{-20} s^{4}-3.866 \times 10^{-15} s^{3}+3.489 \times 10^{-10} s^{2}-2.772 \times 10^{-5} s+1.614}{9.093 \times 10^{-24} s^{5}+1.213 \times 10^{-18} s^{4}+9.768 \times 10^{-14} s^{3}+2.262 \times 10^{-9} s^{2}+7.054 \times 10^{-5} s+1} \\
G_{y y} & =\frac{1.669 \times 10^{-20} s^{4}-2.138 \times 10^{-15} s^{3}+1.891 \times 10^{-10} s^{2}-1.368 \times 10^{-5} s+0.7039}{5.944 \times 10^{-24} s^{5}+7.388 \times 10^{-19} s^{4}+6.959 \times 10^{-14} s^{3}+1.744 \times 10^{-9} s^{2}+7.076 \times 10^{-5} s+1}
\end{aligned}
$$



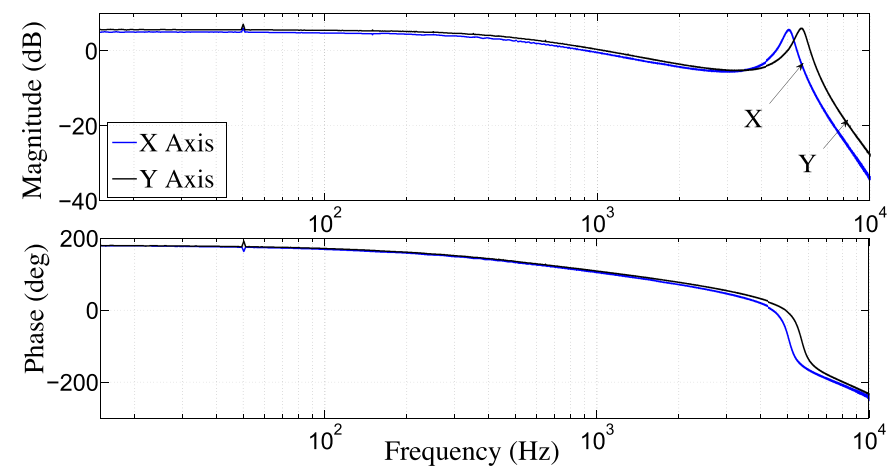

Fig. 10. Closed-loop frequency response comparison between the $x$ - and $y$-axes.

TABLE II

Comparison Between the Position of the Lightly Damped Poles BEFORE AND AFTER THE CONTROL LOOP IMPLEMENTATION

\begin{tabular}{|c|c|c|}
\hline & X Axis & Y Axis \\
\hline \hline Open-loop & $-198 \pm 27873 i$ & $-214.3 \pm 33530 i$ \\
\hline Closed-loop & $-4094.3 \pm 30850.4 i$ & $-2057.9 \pm 35310.6 i$ \\
\hline
\end{tabular}

is also robust to moderate changes in the plant characteristics, and is effective for both axes of the nanopositioner.

A static gain is not typically an effective damping controller for a collocated highly resonant plant with a displacement sensor. However, the bandwidth limited nature of electrothermal sensors and the amplifier results in the open-loop phase response of the system to be close to $-180^{\circ}$ at resonance, which mimics a resonant plant with a velocity sensor. Thus, the damping loop with a proportional controller functions almost as a velocity feedback controller, which is known to be very effective in augmenting damping to a highly resonant structure.

The feedback loop was digitally implemented using a dSPACE-1103 rapid prototyping system, with a sampling rate of $60 \mathrm{kHz}$. Compensator gains of -0.4 and -0.89 were chosen for the $x$ - and $y$-axes, respectively. In Fig. 10, the measured frequency responses of the closed-loop systems are shown (excluding the prefilter in Fig. 9). An improvement of $\sim 30 \mathrm{~dB}$ can be observed and the oscillatory poles are shifted toward the left hand side (Table II). Gain and phase margins are approximately $3.5 \mathrm{~dB}$ and $61.5^{\circ}$.

\section{A. Resolution and Noise Performance}

Under closed-loop conditions, the measurement noise (depicted as $n$ in Fig. 9) is fed back into the system and ultimately affects the positioning accuracy of the nanopositioner. The noise component of the stage displacement $(d)$, known as projected noise [45], is estimated by passing the noise source signal through the closed-loop transfer function from the electrothermal sensor noise $(n)$ to the system output (d) expressed as

$$
T_{\mathrm{dn}}=\frac{d}{n}=\frac{-K C_{\mathrm{sys}}}{1+K C_{\mathrm{sys}}}
$$



(a)

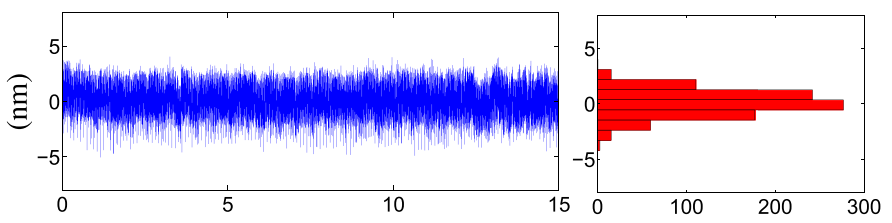

(b)



(c)
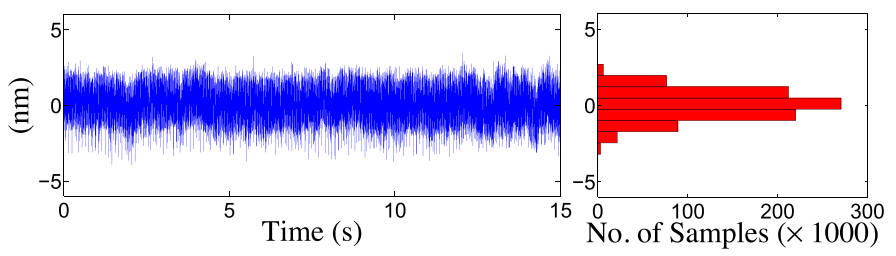

(d)

Fig. 11. Time history of measured noise and the projected noise for the $x$-direction in (a) open loop and (b) closed loop. (c) and (d) Corresponding data for the $y$-axis in open loop and closed loop, respectively. The standard deviation $(\sigma)$ and histogram are also provided for each set of noise data.

In (4), $C_{\text {sys }}$ represents transfer function of the open-loop system in the $x$ - or $y$-direction.

Sensor noises were measured and recorded with $128 \mathrm{kHz}$ sampling rate, whereas all electrostatic comb structures and the scan table were connected to electrical ground. All experiments were performed on a vibration isolated optical table to minimize the effect of external mechanical disturbances on the nanopositioner. The response of the noise transfer function $\left(T_{\mathrm{dn}}\right)$ to the recorded sensor noise signal was then simulated using Simulink. The measured data and the histograms of the sensor noise signals (open-loop noise) and the projected noise in the closed-loop systems for both axes are shown in Fig. 11.

An estimate of the closed-loop displacement resolution is the standard deviation $(\sigma)$ of the projected noise which is about 1.26 and $0.9 \mathrm{~nm}$ for the $x$ - and $y$-axes, respectively. Further increase in displacement resolution is possible by implementing controllers, such as those based on the signal transformation approach (STA) or impulsive state multiplication (ISM) [45], [46].

\section{PRefilter Implementation AND IMAGing}

The controlled nanopositioner was used as the scanning stage of an AFM. Although nonraster scan methods based 


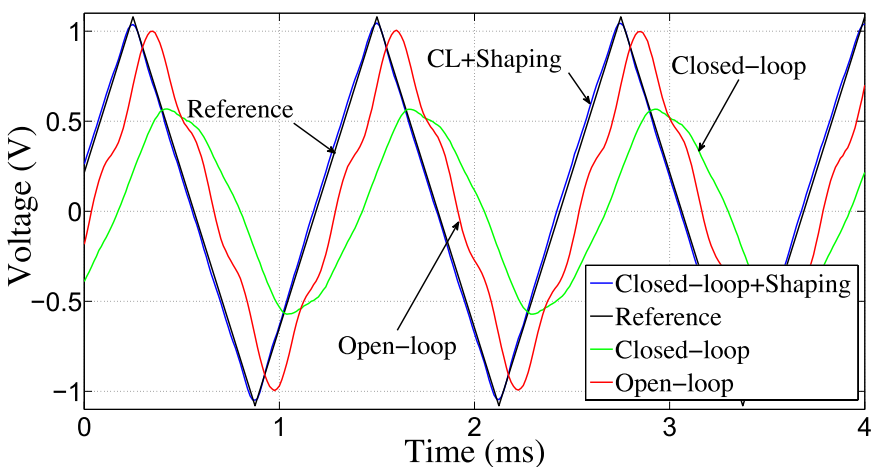

Fig. 12. Tracking performance of the closed-loop system with the input triangular reference signal of $1.08 \mathrm{~V}$ amplitude corresponding to an $x$-axis displacement of $\pm 1 \mu \mathrm{m}$ with $800 \mathrm{~Hz}$.

on spiral, cycloid, and Lissajous patterns exist [47]-[49], that allow for high scan speeds, we decided to first test the performance of the system under traditional rastering conditions. In this method, a triangular signal is applied to one axis (fast axis) and the perpendicular axis is forced to track a slow ramp.

The maximum scan frequency depends on the nanopositioner bandwidth. Under open-loop conditions, the maximum scan speed is $\sim 100$ times lower than the first resonance frequency of the nanopositioner [50]. The additional damping provided by the feedback controller allows for tracking of higher frequency triangular signals as required in high-speed scans. To improve the tracking performance of the fast axis, the reference shaping method, shown in Fig. 9, is used. In this method, the closed-loop transfer function is inverted and implemented as a prefilter [41]. The inversion was implemented offline, by adding the stable steady-state responses of the prefilter transfer function $\left(G_{\mathrm{CL}}^{-1}\right)$ to the Fourier series terms of the triangular reference signal within a bandwidth of $9 \mathrm{kHz}$. The prefilter transfer function for the $x$-axis is described by

$$
G_{\mathrm{CL}}^{-1}=\frac{1+K G_{\mathrm{xx}}}{K G_{\mathrm{Xx}}} .
$$

For each large signal test, the offline prefilter output is experimentally scaled to minimize the error signal. This adjustment is usually less than $\pm 10 \%$ of the closed-loop dc gain obtained by the small signal frequency response.

In Fig. 12, the system's response to an $800-\mathrm{Hz}$ triangular signal with a peak-to-peak displacement of $2 \mu \mathrm{m}$, in open loop, closed loop, and closed loop with reference shaping are compared. For a travel range of $2 \mu \mathrm{m}$, the rms value of the tracking error for open loop, closed loop, and closed loop with shaping are $17.2 \%, 29.7 \%$, and $1.6 \%$, respectively. Clearly, the closed-loop controller with reference shaping method increases the tracking performance drastically.

Although the controller used in this paper has a simple structure, the resulting control performance is comparable with the state-of-the-art control methods for tracking of triangular references, such as STA [45] and ISM [46]. Table III summarizes the performances of these control methods. It can be observed that the approach taken in this paper achieves a considerably small transient time with an
TABLE III

COMPARISON OF CONTROLlER PERFORMANCE IN TRACKING OF Triangular REFERENCES AS REPORTED IN This PAPER, AND Two RECENT MeThods SuItable FOR TRACKING OF TRIANGULAR WAVEForms. THE PEAK-TO-PEAK VALUE OF THE REFERENCE AMPLITUdE IS REPORTED, AND THE ERROR IS DEFINED AS THE PERCENTAGE OF PEAK-TO-PEAK OF THE TraCKING ERror TO PEAK-TO-PEAK OF THE REFERENCE AMPLITUdE

\begin{tabular}{|c|c|c|c|}
\hline Method & $\begin{array}{c}\text { STA } \\
{[45]}\end{array}$ & $\begin{array}{c}\text { ISM } \\
{[46]}\end{array}$ & $\begin{array}{c}\text { Ref. shaping } \\
\text { (current work) }\end{array}$ \\
\hline \hline Open-loop bandwidth $(\mathrm{Hz})$ & 500 & 1600 & 4500 \\
\hline Ref. Frequency $(\mathrm{Hz})$ & 10 & 130 & 800 \\
\hline Ref. Amplitude (P-P) $(\mu \mathrm{m})$ & 10 & 0.8 & 2 \\
\hline Bandwidth (after damping) $(\mathrm{Hz})$ & 200 & 1000 & 700 \\
\hline Error & $6 \%$ & $25 \%$ & $5 \%$ \\
\hline Settling Time $(\mathrm{ms})$ & 700 & 140 & 5 \\
\hline
\end{tabular}

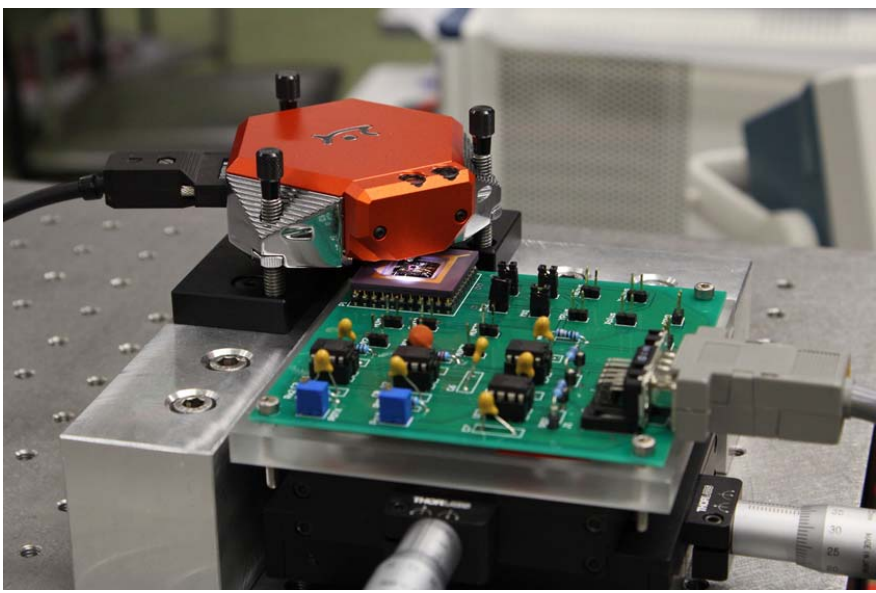

Fig. 13. Setup for testing the MEMS nanopositioner performance under Nanosurf easyscan2 AFM. Actuation and sensor readout circuits are implemented on the PCB shown in the photo.

acceptable tracking error for a fast triangular reference, with a high amplitude.

To examine the performance of the nanopositioner as the scanning stage of an AFM, the gold features fabricated on the scan table, and shown in Fig. 1, were scanned by a Nanosurf easyscan2 AFM. The setup is shown in Fig. 13. The features have a height of $\sim 500 \mathrm{~nm}$ and a spacing of $3 \mu \mathrm{m}$. During the test, the nanopositioner scan table moves under the AFM cantilever. The built-in positioning controller for the $z$-axis stage of the cantilever in the Nanosurf easyscan2 AFM was bypassed to avoid its low bandwidth, and the AFM was operated in constant height contact mode.

For the scan range of $8 \mu \mathrm{m} \times 8 \mu \mathrm{m}$, three scan frequencies of 8,100 , and $200 \mathrm{~Hz}$ were tested. The obtained images are shown in Fig. 14.

The images were obtained using the lateral sensor signals and the $z$-axis signal measuring directly the deflection of the cantilever. The image resolution is $150 \times 150$ pixels, and a single frame was captured in $18.75,1.5$, and $0.75 \mathrm{~s}$ for 8,100 , and $200 \mathrm{~Hz}$ scan frequencies, respectively.

The resonance frequency of the AFM cantilever is $\sim 13 \mathrm{kHz}$. We observe that at the scan frequency of $200 \mathrm{~Hz}$, the cantilever 


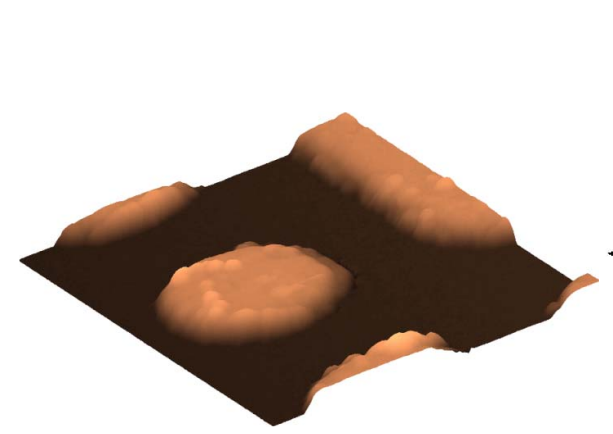

(a)

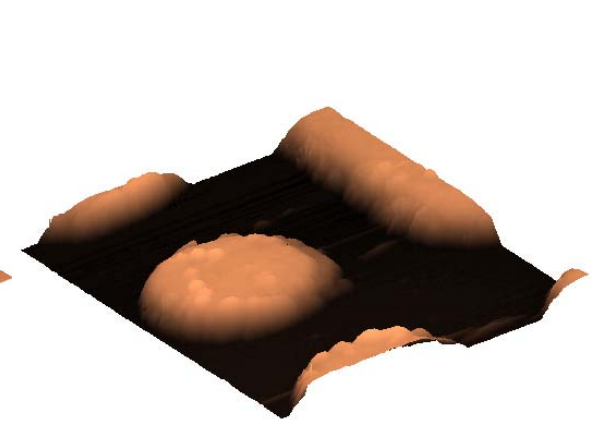

(b)

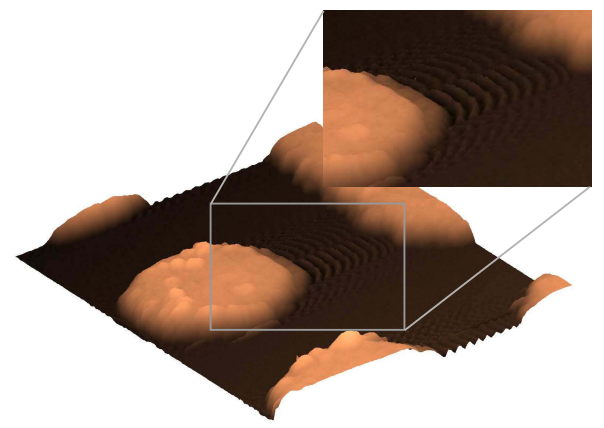

(c)

Fig. 14. 3-D topography of the sample obtained by the AFM at (a) $8 \mathrm{~Hz}$, (b) $100 \mathrm{~Hz}$, and (c) $200 \mathrm{~Hz}$. At the $200 \mathrm{~Hz}$ raster frequency, artifacts due to the cantilever vibrations are shown in close-up view.

starts to vibrate when it undergoes a sharp transition in height. Therefore, in spite of the capability of the nanopositioner to scan at higher rates, higher frequency scans were not attempted. Although this should be possible using cantilevers with higher resonance frequencies.

\section{CONCLUSION}

The design, characterization, and control of a parallel kinematic MEMS nanopositioner with a bandwidth of about $5 \mathrm{kHz}$ was discussed in this paper. Dimensions of the scan table are $1.6 \mathrm{~mm} \times 1.6 \mathrm{~mm}$ and in-plane lateral displacements of about $\pm 5 \mu \mathrm{m}$ were achieved with the cross coupling rejection of $-40 \mathrm{~dB}$. Electrothermal sensors are incorporated to measure lateral displacements of the stage. Exploiting the bidirectional structure of the comb drives, a differential actuation mechanism was implemented that significantly linearized the actuation characteristics of the device. To alleviate the lightly damped behavior of the stage, a closed-loop feedback controller was implemented and characterized. The nanopositioner was operated under an AFM in constant height contact mode. To improve the tracking performance, an off-line inversionfeedforward controller was added to the fast axis of the scanner. The imaging of a set of features fabricated on the scan table was performed with frequencies up to $200 \mathrm{~Hz}$. Although the nanopositioner is capable of achieving much higher scan frequencies, the imaging speed was not increased any further, to prevent damaging the AFM cantilever. We note that the bottle-neck to achieve a video-rate AFM using this nanopositioner is the $z$-axis bandwidth.

\section{REFERENCES}

[1] H. Lan, Y. Ding, H. Liu, and B. Lu, "Review of the wafer stage for nanoimprint lithography," Microelectron. Eng., vol. 84, no. 4, pp. 684-688, Jan. 2007.

[2] G. Binnig and H. Rohrer, "Scanning tunneling microscopy," IBM J. Res. Develop., vol. 44, nos. 1-2, pp. 279-293, 2000.

[3] G. Schitter, K. J. Astrom, B. E. DeMartini, P. J. Thurner, K. L. Turner, and P. K. Hansma, "Design and modeling of a high-speed AFM-scanner," IEEE Trans. Control Syst. Technol., vol. 15, no. 5, pp. 906-915, Sep. 2007.

[4] A. Pantazi et al., "Control of MEMS-based scanning-probe datastorage devices," IEEE Trans. Control Syst. Technol., vol. 15, no. 5, pp. 824-841, Sep. 2007.

[5] M. A. Lantz, H. E. Rothuizen, U. Drechsler, W. Haberle, and M. Despont, "A vibration resistant nanopositioner for mobile parallelprobe storage applications," J. Microelectromech. Syst., vol. 16, no. 1, pp. 130-139, Feb. 2007.
[6] E. Eleftheriou, "Nanopositioning for storage applications," Annu. Rev. Control, vol. 36, no. 2, pp. 244-254, Dec. 2012.

[7] Y. K. Yong, S. S. Aphale, and S. O. R. Moheimani, "Design, identification, and control of a flexure-based XY stage for fast nanoscale positioning," IEEE Trans. Nanotechnol., vol. 8, no. 1, pp. 46-54, Jan. 2009.

[8] Y. K. Yong, S. O. R. Moheimani, B. J. Kenton, and K. K. Leang, "Invited review article: High-speed flexure-guided nanopositioning: Mechanical design and control issues," Rev. Sci. Instrum., vol. 83, no. 12, p. 121101, Dec. 2012.

[9] T. Ando, "High-speed atomic force microscopy coming of age," Nanotechnology, vol. 23, no. 6, p. 062001, 2012.

[10] J. P. Howard-Knight and J. K. Hobbs, "Video rate atomic force microscopy using low stiffness, low resonant frequency cantilevers," Appl. Phys. Lett., vol. 93, no. 10, pp. 104101-1-104101-3, 2008.

[11] B. J. Kenton and K. K. Leang, "Design and control of a three-axis serial-kinematic high-bandwidth nanopositioner," IEEE/ASME Trans. Mechatronics, vol. 17, no. 2, pp. 356-369, Apr. 2012.

[12] K. K. Leang and A. J. Fleming, "High-speed serial-kinematic SPM scanner: Design and drive considerations," Asian J. Control, vol. 11, no. 2, pp. 144-153, Mar. 2009.

[13] Y. K. Yong, K. Liu, and S. O. R. Moheimani, "Reducing cross-coupling in a compliant $\mathrm{XY}$ nanopositioner for fast and accurate raster scanning," IEEE Trans. Control Syst. Technol., vol. 18, no. 5, pp. 1172-1179, Sep. 2010.

[14] A. G. Fowler, A. N. Laskovski, A. C. Hammond, and S. O. R. Moheimani, "A 2-DOF electrostatically actuated MEMS nanopositioner for on-chip AFM," J. Microelectromech. Syst., vol. 21, no. 4, pp. 771-773, Aug. 2012.

[15] E. Guliyev et al., "Quasi-monolithic integration of siliconMEMS with piezoelectric actuators for high-speed non-contact atomic force microscopy," Meas. Sci. Technol., vol. 23, no. 7, pp. 074012-1-074012-8, 2012.

[16] S. S. Aphale, B. Bhikkaji, and S. O. R. Moheimani, "Minimizing scanning errors in piezoelectric stack-actuated nanopositioning platforms," IEEE Trans. Nanotechnol., vol. 7, no. 1, pp. 79-90, Jan. 2008.

[17] P. Vettiger et al., "The 'millipede'-Nanotechnology entering data storage," IEEE Trans. Nanotechnol., vol. 1, no. 1, pp. 39-55, May 2002.

[18] E. Eleftheriou et al., "Millipede-A MEMS-based scanning-probe datastorage system," IEEE Trans. Magn., vol. 39, no. 2, pp. 938-945, Mar. 2003.

[19] J. B. C. Engelen et al., "A mass-balanced through-wafer electrostatic $x / y$ scanner for probe data storage," Microelectron. Eng., vol. 86, nos. 4-6, pp. 1230-1233, 2009.

[20] A. Mohammadi, A. G. Fowler, Y. K. Yong, and S. O. R. Moheimani, "A feedback controlled MEMS nanopositioner for on-chip high-speed AFM," IEEE J. Microelectromech. Syst., vol. 23, no. 3, pp. 610-619, Jun. 2014.

[21] Y. Zhu, A. Bazaei, S. O. R. Moheimani, and M. R. Yuce, "Design, modeling, and control of a micromachined nanopositioner with integrated electrothermal actuation and sensing," J. Microelectromech. Syst., vol. 20, no. 3, pp. 711-719, 2011.

[22] R. Hickey, D. Sameoto, T. Hubbard, and M. Kujath, "Time and frequency response of two-arm micromachined thermal actuators," J. Micromech. Microeng., vol. 13, no. 1, pp. 40-46, 2003.

[23] C. Guan and Y. Zhu, "An electrothermal microactuator with Z-shaped beams," J. Micromech. Microeng., vol. 20, no. 8, p. 085014, 2010. 
[24] A. Cowen, G. Hames, D. Monk, S. Wilcenski, and B. Hardy, SOIMUMPs Design Handbook. Revision 8.0, 2011, MEMSCAP Inc.

[25] M. Maroufi and R. S. O. Moheimani, "Design, fabrication and characterization of a high-bandwidth 2DOF MEMS nanopositioner," in Proc. IEEE/ASME Int. Conf. Adv. Intell. Mechatron., Jul. 2013, pp. 335-340.

[26] E. P. Popov and T. A. Balan, Engineering Mechanics of Solids. Englewood Cliffs, NJ, USA: Prentice-Hall, 1998.

[27] R. Legtenberg, A. W. Groeneveld, and M. Elwenspoek, "Comb-drive actuators for large displacements," J. Micromech. Microeng., vol. 6, no. 3, pp. 320-329, 1996.

[28] D. C. Miller, B. L. Boyce, M. T. Dugger, T. E. Buchheit, and K. Gall, "Characteristics of a commercially available silicon-on-insulator MEMS material," Sens. Actuators A, Phys., vol. 138, no. 1, pp. 130-144, Jul. 2007.

[29] Y.-S. Kim, J.-M. Yoo, S. H. Yang, N. G. Choi, Y.-M. Dagalakis, and S. K. Gupta, "Design, fabrication and testing of a serial kinematic MEMS $X Y$ stage for multifinger manipulation," J. Micromech. Microeng., vol. 22, no. 8, p. 085029, 2012.

[30] F. Khan, S. A. Bazaz, and M. Sohail, "Design, implementation and testing of electrostatic SOI MUMPs based microgripper," Microsyst. Technol., vol. 16, no. 11, pp. 1957-1965, 2010.

[31] Y.-C. Chen, I. C.-M. Chang, R. Chen, and M. T.-K. Hou, "On the side instability of comb-fingers in MEMS electrostatic devices," Sens. Actuators A, Phys., vol. 148, no. 1, pp. 201-210, Nov. 2008.

[32] A. G. Fowler, A. Bazaei, and S. O. R. Moheimani, "Design and analysis of nonuniformly shaped heaters for improved MEMS-based electrothermal displacement sensing," J. Microelectromech. Syst., vol. 22, no. 3, pp. 687-694, Jun. 2013.

[33] A. Mohammadi, M. R. Yuce, and S. O. R. Moheimani, "A lowflicker-noise MEMS electrothermal displacement sensing technique," J. Microelectromech. Syst., vol. 21, no. 6, pp. 1279-1281, Dec. 2012.

[34] Y. K. Yong, A. G. Fowler, A. Mohammadi, and S. O. R. Moheimani, "Control of a MEMS nanopositioner for atomic force microscopy," in Proc. 6th IFAC Symp. Mechatron. Syst., Hangzhou, China, Apr. 2013 pp. 375-382.

[35] D. Sidobre and V. Hayward, "Calibrated measurement of the behaviour of mechanical junctions from micrometre to subnanometre scale: The friction force scanner," Meas. Sci. Technol., vol. 15, no. 2, pp. 451-459, 2004.

[36] B. Bhushan, Springer Handbook of Nanotechnology, 2nd ed. New York, NY, USA: Springer-Verlag, 2007

[37] M. A. Soderstrand, G. Berchin, and R. S. Roberts, "Frequency domain least-squares system identification," Int. J. Electron., vol. 78, no. 1, pp. 25-35, 1995.

[38] U. Durig, "Fundamentals of micromechanical thermoelectric sensors," J. Appl. Phys., vol. 98, no. 4, pp. 044906-1-044906-14, Aug. 2005.

[39] S. S. Aphale, A. J. Fleming, and S. O. R. Moheimani, "Integral resonant control of collocated smart structures," Smart Mater. Struct., vol. 16, no. 2, p. 439, 2007.

[40] B. Bhikkaji, M. Ratnam, A. J. Fleming, and S. O. R. Moheimani, "Highperformance control of piezoelectric tube scanners," IEEE Trans. Control Syst. Technol., vol. 15, no. 5, pp. 853-866, Sep. 2007.

[41] B. Bhikkaji, M. Ratnam, and S. O. R. Moheimani, "PVPF control of piezoelectric tube scanners," Sens. Actuators A, Phys., vol. 135, no. 2, pp. 700-712, Apr. 2007.

[42] B. Bhikkaji, S. O. R. Moheimani, and I. Petersen, "A negative imaginary approach to modeling and control of a collocated structure," IEEE/ASME Trans. Mechatronics, vol. 17, no. 4, pp. 717-727, Aug. 2012.

[43] A. Lanzon and I. Petersen, "Stability robustness of a feedback interconnection of systems with negative imaginary frequency response," IEEE Trans. Autom. Control, vol. 53, no. 4, pp. 1042-1046, May 2008.

[44] I. R. Petersen, "Negative imaginary systems theory in the robust control of highly resonant flexible structures," in Proc. Austral. Control Conf. (AUCC), Nov. 2011, pp. 1-6.

[45] A. Bazaei, Y. K. Yong, S. O. R. Moheimani, and A. Sebastian, "Tracking of triangular references using signal transformation for control of a novel AFM scanner stage," IEEE Trans. Control Syst. Technol., vol. 20, no. 2, pp. 453-464, Mar. 2012.

[46] T. Tuma, A. Pantazi, J. Lygeros, and A. Sebastian, "Nanopositioning with impulsive state multiplication: A hybrid control approach," IEEE Trans. Control Syst. Technol., vol. 21, no. 4, pp. 1352-1364, Jul. 2013.

[47] I. A. Mahmood and S. O. R. Moheimani, "Fast spiral-scan atomic force microscopy," Nanotechnology, vol. 20, no. 36, p. 365503, 2009.

[48] Y. K. Yong, S. O. R. Moheimani, and I. R. Petersen, "High-speed cycloid-scan atomic force microscopy," Nanotechnology, vol. 21, no. 36, p. $365503,2010$.
[49] A. Bazaei, Y. K. Yong, and S. O. R. Moheimani, "High-speed Lissajousscan atomic force microscopy: Scan pattern planning and control design issues," Rev. Sci. Instrum., vol. 83, no. 6, p. 063701, 2012.

[50] S. Devasia, E. Eleftheriou, and S. O. R. Moheimani, "A survey of control issues in nanopositioning," IEEE Trans. Control Syst. Technol., vol. 15, no. 5 , pp. $802-823$, Sep. 2007.

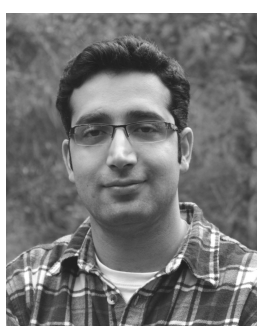

Mohammad Maroufi received the B.Sc. degrees in mechanical engineering and applied physics and the M.Sc. degree in mechatronics from the Amirkabir University of Technology, Tehran, Iran, in 2008 and 2011, respectively. He is currently pursuing the Ph.D. degree in electrical engineering with the University of Newcastle, Callaghan, NSW, Australia.

His current research interests include design and control of MEMS nanopositioning systems, MEMSbased sensing and actuation, on-chip atomic force microscopy, and mechanical modeling of smart materials and structures.

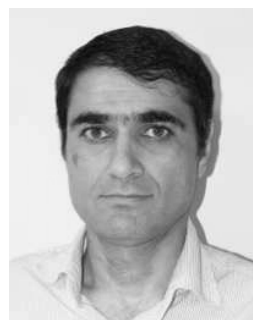

Ali Bazaei (M'10) received the B.Sc. and M.Sc. degrees in electrical engineering from Shiraz University, Shiraz, Iran, in 1992 and1995, respectively, and the Ph.D. degrees in electrical engineering from Tarbiat Modares University, Tehran, Iran, and the University of Western Ontario, London, ON, Canada, in 2004 and 2009, respectively.

He was an Instructor with Yazd University, Yazd, Iran, from 1995 to 2000, and a Research Assistant with the Department of Electrical and Computer Engineering, University of Western Ontario, from 2004 to 2005. He is currently a Research Academic with the School of Electrical Engineering and Computer Science, University of Newcastle, Callaghan, NSW, Australia. His current research interests include nonlinear systems, including control and modeling of structurally flexible systems, friction modeling and compensation, neural networks, and microposition sensors.

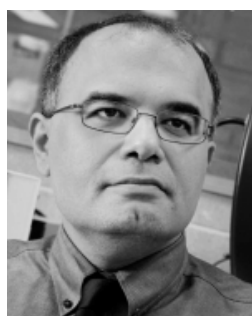

S. O. Reza Moheimani (M'96-SM'00-F'11) received the Ph.D. degree from the University of New South Wales, Australian Defence Force Academy, Canberra, ACT, Australia, in 1996.

He joined the University of Newcastle, Callaghan, NSW, in 1997, where he embarked on a new research program addressing the dynamics and control design issues related to high-precision mechatronic systems. He is the Founder and the Director of the Laboratory for Dynamics and Control of Nanosystems, a multimillion-dollar state-of-the-art research facility. He has authored over 300 refereed papers, five books, and edited volumes. His current research interests include ultrahigh-precision mechatronic systems, with particular emphasis on dynamics and control at the nanometer scale, including applications of control and estimation in nanopositioning systems for high-speed scanning probe microscopy, modeling and control of microcantilever-based devices, control of microactuators in microelectromechanical systems, and design, modeling, and control of micromachined nanopositioners for on-chip atomic force microscopy.

Prof. Moheimani is a fellow of the International Federation of Automatic Control (IFAC) and the Institute of Physics, U.K. He is currently the Chair of the IFAC Technical Committee on Mechatronic Systems, and has been the Chair of several international conferences and workshops. He has served on the Editorial Boards of a number of journals, including the IEEE/ASME TRANSACTIONS ON MECHATRONICS, the IEEE TRANSACTIONS ON CONTrol Systems Technology, and Control Engineering Practice. He was a recipient of a number of awards, including the IFAC Nathaniel B. Nichols Medal in 2014, the IFAC Mechatronic Systems Award in 2013, the IEEE Control Systems Technology Award in 2009, the Australian Research Council Future Fellowship in 2009, the IEEE TRANSACTIONS ON CONTROL SYSTEMS TEChNOLOGY Outstanding Paper Award in 2007, and the Australian Research Council Post-Doctoral Fellowship in 1999, and received several best student paper awards in various conferences. 